\title{
An Efficient Data Transmission by Using Modern USB Flash Drive
}

\author{
B. Naresh Kumar Reddy, N. Venktram and T. Sireesha \\ Dept of E. C. M, K. L.University,Guntoor(dt),India \\ naresh.klu@gmail.com,bnkreddy@kluniversity.in,venktram@kluniversity.in
}

\begin{abstract}
Now a day's Electronics plays a vital role in our daily life. It is a key in making this world a small and making everything happen at our finger tips, today's most used and flexible is USB flash drives, which is used to store the data and data transfer between them related with computer, and we are not able to share files between two USB flash driveswhen user is away from computer. So to overcome this problem we are designing a complete blend of hardware and software With the help of this project we can not only transfer the data but also we can see the transfer of the particular file which we want to send by using LCD display. In our proposal we are transferring the data between twe USB flash drives using two ways. One is Wireless Technology either Wi-Fi technology which we cansend/receive File or Bluetooth Technology which we can send/receive data to USB flash drive with Bluetooth enabled devices like Mobiles, laptops etc.. Another is in-bult USB slots to perform data transmission to other pen drives directly without the use of the computer system. The implementation of an operating system and a processon to the USB Flash drive are the main challenges in this proposed system.
\end{abstract}

Keywords: Wi-Fi, Bluetooth, USB Flash drive, micro sd card

\section{Introduction}

Today's clients are pulled in with a wide mixture of remote innovations, frameworks, and merchants to address their needs for remote advances. Besides, the greater part of clients will find that none of one remote innovation result is suitable for all requisitions. Case in point, low-information rate informing might be served by the numerous

Open alternatives for two-way paging and narrowband Particular Correspondence Framework. For higher information rates, Remote LANs offer a great answer for a nearby area [1-2]. While clients may select from numerous remote information administrations, a lot of people nore clients pick a Remote LAN framework to meet information necessities. Remote CAN supply rapid, dependable information, correspondences in a yard environment and scope in little zones. Remote LANs are easy to introduce and don't cause month to month client information transmission charges.

\section{A. Wi-Fi}

Wi-Fi implies remote devotion, it is a most loved engineering that permits an electronic gadget to trade information to the web without utilizing of wires by using radio waves. But the extent of wifi is limited. a ordinary wireless access point utilizing $802.11 \mathrm{~b}$ or $802.11 \mathrm{~g}$ with a stock antenna strength have a reach of $35 \mathrm{~m}(120 \mathrm{ft})$ inside and $100 \mathrm{~m}(300 \mathrm{ft})$ outdoors [3].

Usage: Wi-Fi employments: Wi-Fi is progressively turned into the vital mode of web association throughout the world. To get to wifi association, one must have a remote 
connector on their machine. Wi-Fi gives remote connectivity by serving frequencies between $2.4 \mathrm{GHz}$ to $5 \mathrm{GHz}$ focused around the measure of information rate on the network [3-4].

The Wi-Fi connectivity empowers zones known as Problem areas. Here we utilized progressed programming similar to Remote on to discover and solicitation association with Problem areas. To begin a Remote association, first the remote switch is connected to the web association and after that all the obliged settings are appropriately introduced.

Wifi system utilizes the radio waves for the transmission and gathering of the data. before we see that machine obliged remote connector [5], and keen cell additionally obliged utilization wifi equipment same way we plan one exceptional fittings for pen drive it has uncommon processor like SOC(system on chip)and likewise we can introduce the obliged conventions for the Wifi in that processor.

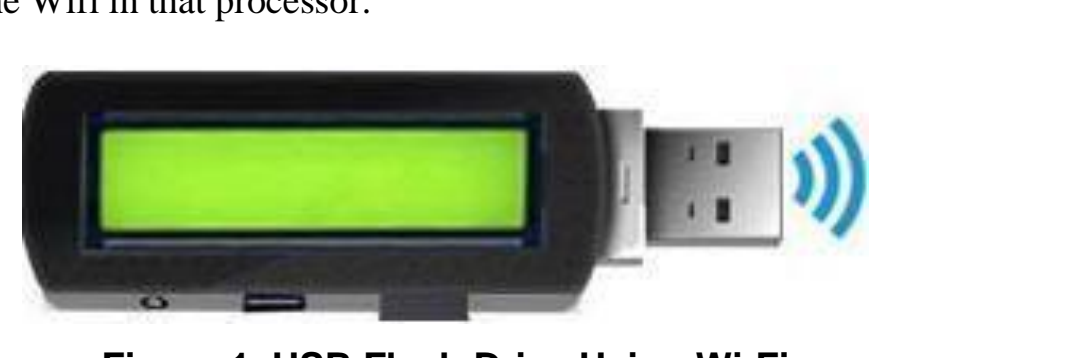

\section{B. Bluetooth}

Figure 1. USB Flash Drive Using Wi-Fi

Bluetooth engineering is a link Tade, innovation intended for remote association with peripherals, like as mice and cell telephones $x$ to your desktop or portable computer to one another. Bluetooth is a bundle based convention with an expert slave structure. One expert can speak with up to 7 slave's whole gadgets impart the expert's clock. Information Parcel trade is focused around the essential clock, given by the expert, which ticks at $312.5 \mu \mathrm{s}$ interims. Two clocks ticks can join to make up an opening of $625 \mu \mathrm{s}$; two spaces can consolidate to make up a space pair of $1250 \mu$ s.for sample in the straightforward instance of single-space bundles the expert transmits in even openings and accepts in odd spaces, the slave, persistently, gets in an even openings and transmits in odd openings. Bundles may be 1, 3,5 or 7 openings in length, however in all cases, the Master transmits will first send even spaces and after that slave transmit odd slots [6-7].

I. The range of Bluetooth is up to 10 meters

II. Here do not require line of sight communication.

This makes Bluetooth communication much more flexible, reliable and robust. Bluetooth excels at low bandwidth data transfer, it is not used as a replacement for high-bandwidth cabled peripherals.

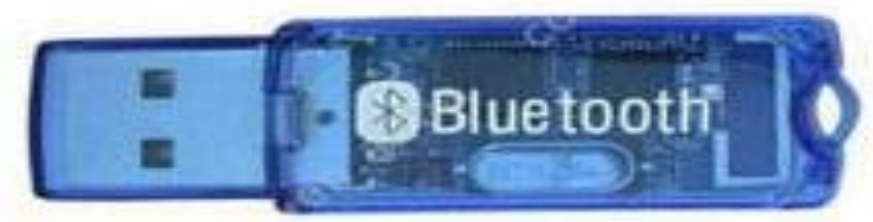

Figure 2. USB Flash Drive using Bluetooth

Bluetooth also required special type protocols, so separate software program is installed in our Flash Drive. 


\section{USB Flash Drive}

A USB flash drive or pen-drive is an information stockpiling gadget that comprises of blaze memory with an incorporated Wide spread Serial Transport (USB) interface. USB blaze drives are regularly removable and re-writable, and physically much more modest than an optical disc [8]. USB Flash drives are habitually utilized for the same purposes for which floppy plates or Compact discs were utilized, i.e., for capacity, move down and exchange of information records. They are more modest, high velocity, and it comprises many times more limits, and is solid in light of the fact that they don't have moving parts. Besides USB blaze drives are resistant to attractive obstruction, and unharmed by surface scratches (dissimilar to Discs). Our principle point likewise we coordinate entire chip in the little pen drive so we plan uncommon processor for this engineering. By and large USB glimmer thive has no additional processor is utilized for preparing yet we might be one unique processor and outline that for this operation [9-10].

\section{USB}

Figure 3. USB Flash Drive using USB Slot

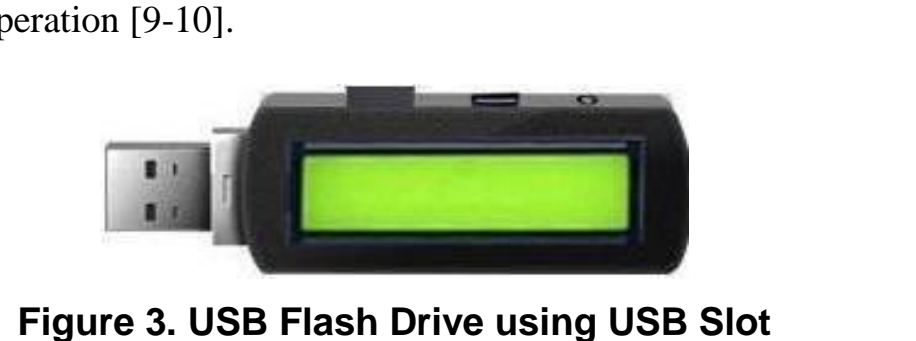

Universal Serial Bus (USB) is an industry standard that lets some know conventions, those are characterizes the links and connectors. These correspondence conventions utilized as a part of association for information, correspondence through transports and likewise utilized for force supply between machines and electronic gadgets Here USB intended for one pen drive insertion to an alternate pen drive. Here additionally USB has any particular principles for association Station Ordinarily ve see USB's are utilized as a part of pc's and laptops in association with alternate peripherals, however here we utilize just pen-drive insertion, so here we utilize some obliged drivers are introduced in our processor [11-12].

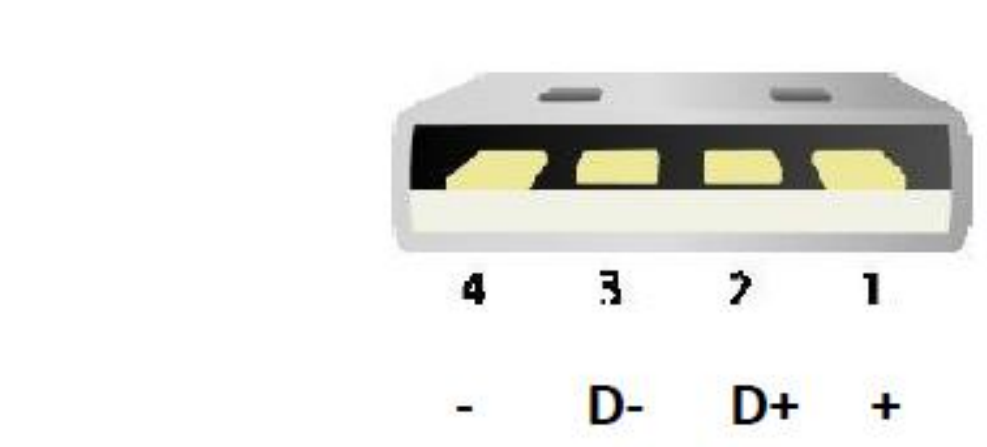

Figure 4. USB Flash Drive Operation

\section{E. A Memory Card}

A memory card is an electronic flash memory data storage device used for storing data. They are very small size, but we can use large data capacity storage. They are normally used in many more electronic devices, digital cameras, mobiles, and laptops. Most of these can be compact in size, re-recordable, and can retain data without power [13]. 


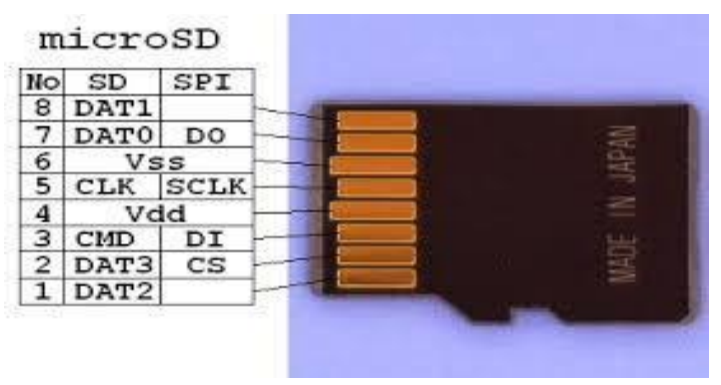

Figure 5. Memory Card

\section{F. LCD}

A liquid crystal display (LCD) is a meager, level showcase gadget made up of any number of shade or monochrome pixels displayed before a light solurce or feflector. Every pixel comprises of a segment of fluid precious stone particles suspended between two transparent cathodes, and two polarizing channels, the tomanawks of extrenity of which are perpendicular to one another. Without the fluid precious stones between them, light passing through one might be hindered by the other. The fluid precious stone turns the polarization of light entering one channel to permit it to pass through theother [13].

A program must interact with the outside world using input and output devices that communicate directly with a human being. One of the most common devices attached to a controller is an LCD display. Probably the most well-known LCDs joined with the controllers are 16x1, 16x2 and 20x2 showcases. This means 16 characters of every line by 1 line 16 characters of every line by 2 lines and 20 characters of every line by 2 lines, respectively [14].

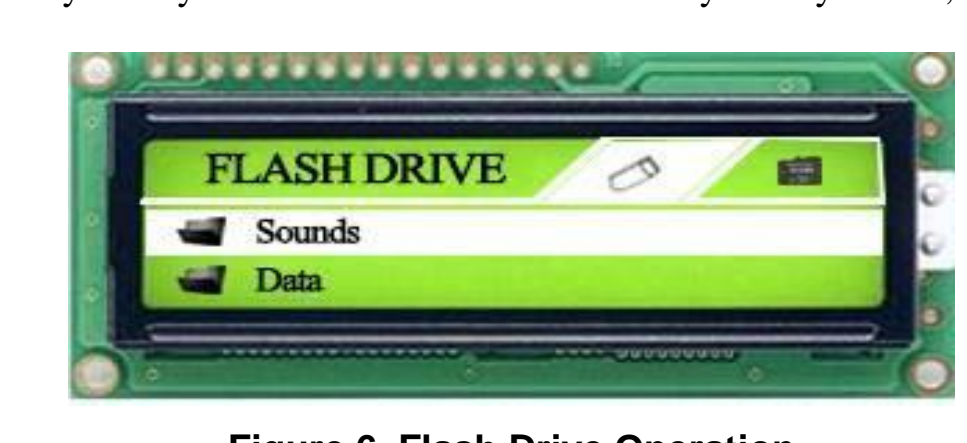

Figure 6. Flash Drive Operation

\section{Requirements of System}

The need of transferring data, in minimum possible time, from one USB Flash Drive to another has become essential after the success of USB Flash Drives or as we say 'pen drives'. It involves a series of processes to achieve a successful transfer when being done with the use of a computer. Starting with locating a working computer system, connecting the Flash Drive to the USB port, and waiting for the computer system to recognize the device after the driver has been installed, to finally selecting the data to be transferred, and ensuring that the transfer had completed. All of this consumes a lot of time and power too, as the computer is required to be totally functional before transferring the data. It is also not feasible to carry around the laptop everywhere with you when there is a much more viable and convenient alternate

In our project we are transferring the data between two USB flash drives using two ways. One is Wireless Technology which we can send the data wireless with help of 
Wi-Fi technology and Bluetooth Technology, and another way is Wired Technology through USB Slots and Memory Card Slots.

Requirement 1: In wireless Method first install Wi-Fi drivers and Bluetooth Drivers, this technology has used which we can data communication to USB flash drive

Requirement 2: In-built USB slots and SD card slots used to perform data communication between one to other pen drives directly without the involvement of the computer system

Requirement 3: Presentation unit - The Display unit is the progressed characteristic in the current pen drives. The presentation of the pen drive could be outlined atilizing touch screen .Utilizing this unit we can see the substance in the pen drive utilizing the touch screen. Utilizing the touch screen within the pen drive with lessen the space involved by the catches in the pen drives. There are numerous chidren of showcase innovations. The capacity tube realistic presentation is the most seasoned innovation utilized for showcase yet now we are utilizing the new virtual help for present day pen drives. Taking into account touch screen information might be transmitting one USB glimmer drive to an alternate USB blaze Drive [17].

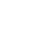

Requirement 4: Charge Terminal - While utilizing the Up to date pen drives if the battery is low we can charge the pen arive by utilizing the cell telephone connector within the charge terminal space. Il requires just little measure of force.

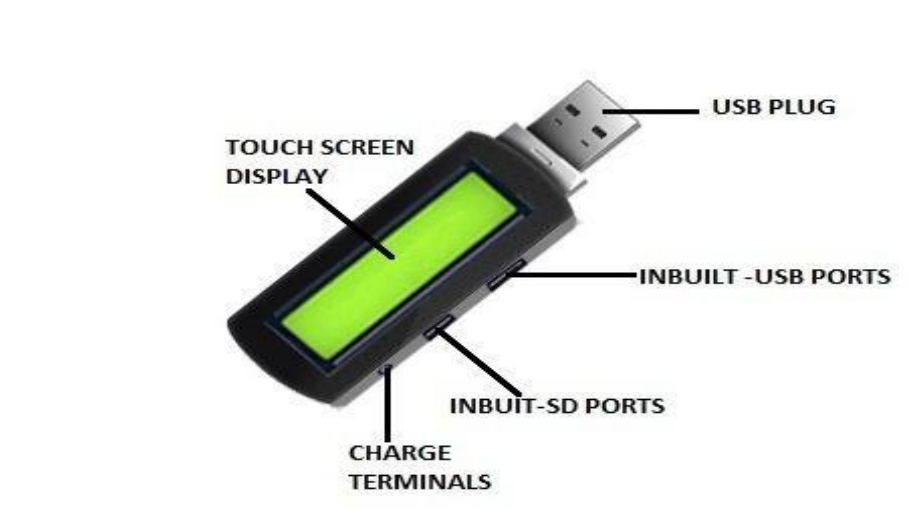

Figure 7. USB Flash Drive Parts

The device will have following features
A. Flash Drive USB 2.0 with Bluetooth chip
B. USB 2.0 with Wi-Fi connector
C. Powered by $9 \mathrm{~V}$ battery
D. Hardware to install Wi-Fi drivers
E. Turn on/off switch for Wi-Fi
F. Touch Screen Display
G. Inbuilt USB ports
H. Enabling pen drive to act as a card reader 


\section{Implementation}

The USB to USB data transfer device consists of the following main parts:

\section{USB2.0:}

USB is an expert slave transport with one expert and various slaves. The expert is known as a host and the slaves are the peripherals, for example, a machine framework can start the information exchanges; the slaves just react to the host's directions they never launch exchanges

The correspondence made by USB is focused around legitimate channels - known as 'funnels'. It unites the host controller to the gadget endpoint. The endpoint is a sensible element which lives onto the gadget the associations made is 1 to 1 for the endpoints in channels. A USB gadget can have 32 endpoints- two of which are held. Remaining 30 are available for ordinary utilization.

The data transfer is having four types:

1. Interrupt transfers: for the devices needing quick but guaranteed response (e.g. pointing device).

2. Isochronous transfers: For some fixed data rate but data loss may take place (e.g. audio, video)

3. Control transfers: used for simple status check.

4. Bulk transfers: uses available bandwidth with no fixed data rate (e.g. file transfer).

The speeds offered by the USB are defined in the USB2.0 specification:
a. $\quad$ Low speed: $1.5 \mathrm{Mbps}$
b. $\quad$ Full speed: 12 Mbps
c. High speed. $480 \mathrm{Mbps}$

\section{A.WI-FI Connector with USB Flash Drive}

Data is tran mitted USB to Another Wi-Fi enabled Devices like as Mobiles, laptops etc at that time we are using Wi-Fi)USB connector in my project using Nintendo Wi-Fi USB Connector

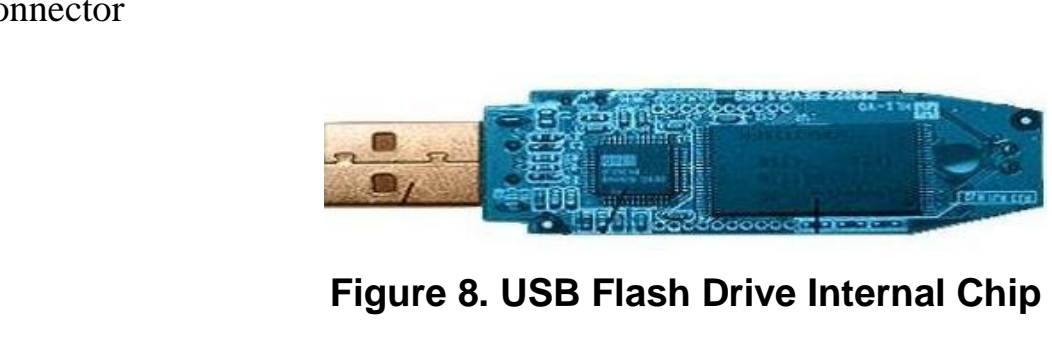

Remote engineering has generally spread of late and you can go anyplace; at home, at work, in libraries, schools, runways, inns and even in a few restaurants.

Remote systems administration is known as Wi-Fi. (Wireless Fidelity) or 802.11 systems administration as it blankets the IEEE 802.11 advances. The real focal point of Wi-Fi is that it is perfect with practically every working framework, diversion gadget and progressed printer Like cell telephones, a Wi-Fi system makes utilization of radio waves to transmit data over a system. The machine ought to incorporate a remote connector that will make an interpretation of information sent into a radio indicator. This same sign will be transmitted, by means of a reception apparatus, to a decoder known as the switch. Once decoded, the information will be sent to the Internet through a wired Ethernet association. As the remote system will act as a 
two-way activity, the information accepted from the Internet will additionally pass through the switch to be coded into a radio indicator that will be receipted by the workstation's remote connector.

\section{B. Bluetooth}

Bluetooth is utilized within our venture as a corresponding gadget with the other Bluetooth empowered gadget to construct a way characterized by ISM band and join it to move the information in the pen drive. Bluetooth engineering has additionally obliged exceptional sort of drivers and fittings, those likewise introduce and run those drivers and access those data [10].

\section{Proposed Block Diagram:}

a. Flash Drive with USB interface: It is the pen drive for keeping the dat stored in it. They are also called as flash drive.

b. Operating System: It is used so that the drivers of Bhetooth and frie manager can be installed easily in it using the operating system.

c. Bluetooth Hardware: It is the Bluetooth chip or device used o create a link between two or more operating devices. Here WT12 Bluetooth chip will be used manufactured by BLUEGIGA Pvt Ltd.

d. Communication Link: It is the hink through which data will travel from other Bluetooth enabled devices to the pen drive or vice versa.

e. Battery: It is used to provide the power to Bluetooth chip and memory drive so that it can perform read/write operation.

f. Driver: It is required to allow the use of Bluetooth over the processor used and also for some data handling process.

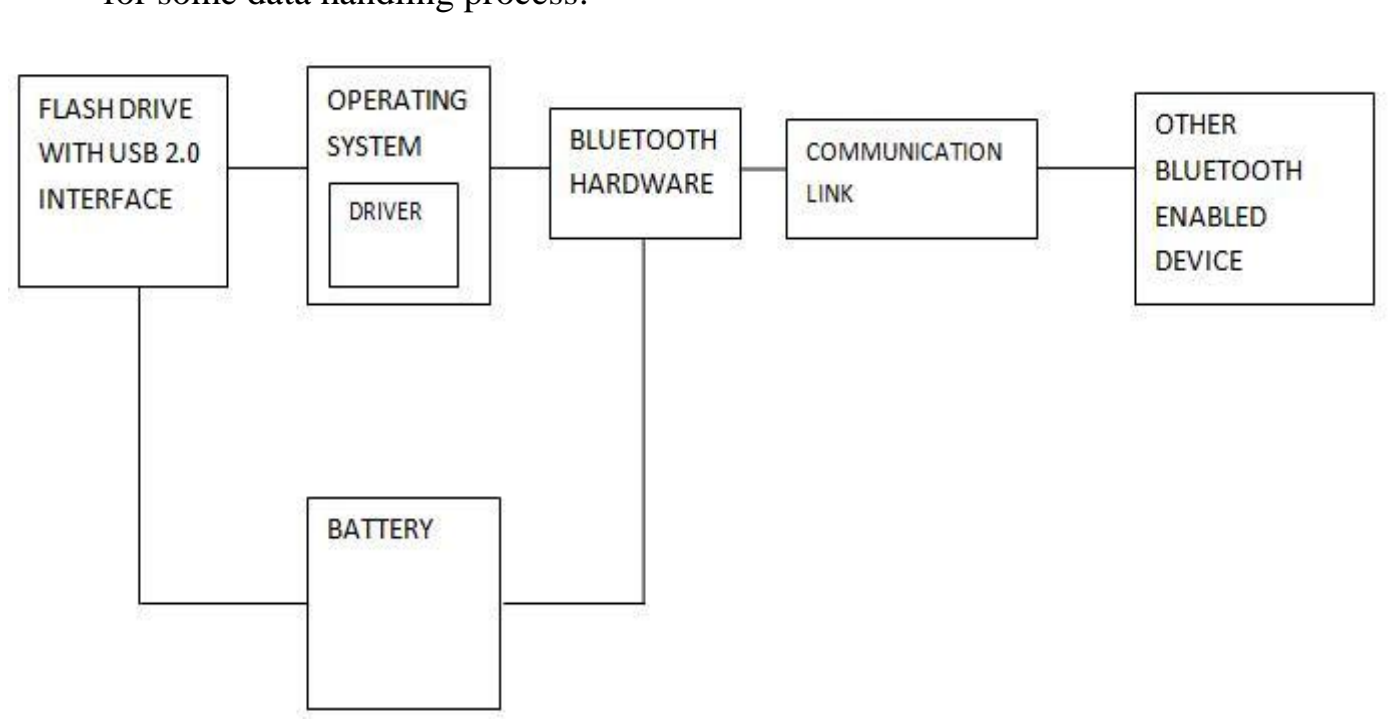

Figure 9. Proposed Block Diagram Bluetooth in USB

\section{In-Built USB Slot:}

In-Built USB slots in USB flash drive, Data can be transmitted or Received USB to Another USB without helping of Computer 


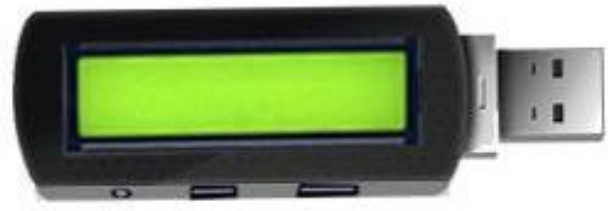

Figure 10. In-built USB Slot in Flash Drive

Its primary characteristics are touch screen, two USB spaces and has charge terminal it work similar to workstation. [15] We can exchange the information from one pendrive to the modernized pen drive and alter the information's to our needs. It is more ptodest, speedier, less expensive and movable. It has the less charge terminal to the Charge as like mart phones and so forth. They have a blaze memory that is easier origination of force by developed microchip .We can even play tunes and features there is a need of conduct dissection of

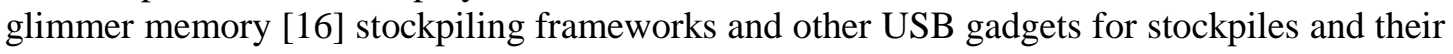
assessments. Specifically, a set of assessment measurements and their relating access examples are proposed. The practices of glimmer memory are likewise examined regarding execution and unwavering quality issues the security of the pen drive could be executed be a few systems which can distinguish particular drive. We can portray the techniques for advanced proof dissection about USB thumb drives oladgets, for example, Computerized Pen Drive. The rate of the pen dirices which is executed with these characteristics can additionally be shabby as the ordinary arives being in the business now this can truly make an element stockpiling and transmission gadget. This proposal when executed has more effect on the pen drive deals. The foca points of utilizing such USB pen drives and blaze drives are principally on the grounds that their energy utilization and vitality overhead characteristics.

The primary preferences are recorded as takes after:

- Very handy and compact

- Display unit to display its contents

- Extendable memory fots

- High speed processor and user friendly OS

- Chargeable battery

- Affordable cost

\section{Data Transmission}

Data transmission one USB Flash Drive to another USB Flash Drive in two ways

\section{A. Wireless Data Transmission}

In this way Data transmission or Reception through Wi-Fi and Bluetooth [17-18].

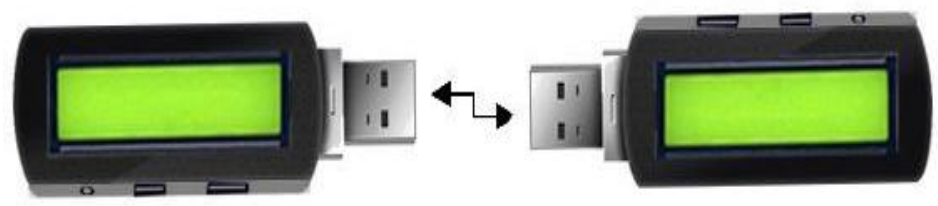

Figure 11. Wireless Data Transmission 


\section{B. USB Slot}

The Data transmission is likewise the propelled characteristic in the automated pen drive. Utilizing this characteristic we can transmit the information to any viable USB gadgets and the transmission from whatever available USB gadgets could be made simple and basic utilizing this modernized pen drive. The working of the automated pen drive is like the workstation framework. The information transmission through this automated pen drive will decrease the utilization of force. The need to join transportable electronic gadgets to one another has quickened the appropriation of USB on-the-go as an Industry standard wired interface for interfacing the two gadgets ideas. The information transmission in the Computerized Pen Drive can likewise be carried out without the utilization of the frameyoork. Information Transmission through USB Slot as indicated as fig underneath

\section{SD Card Slot}

This slot can be used Data transmission or Reception USBDEVICE to Metmory card.

\section{Functionality}

Figure 12. Data Transmission through USB and SD Card Slot

Functionality of this pen drive is very simple like smart phone. When we select the menu option on the touch screen, then two sub options are displayed there are

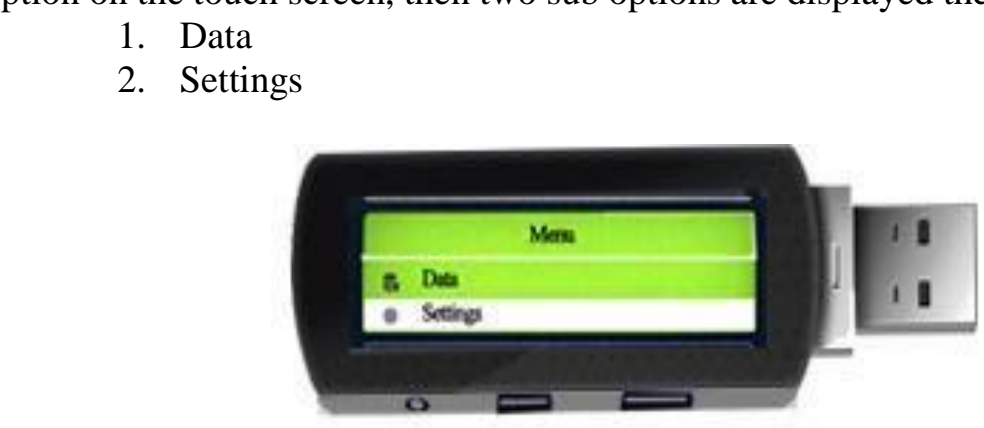

Figure 13. USB Flash Drive Functionality in Menu

The internal working of this function is when we touch menu options the processor reading the request signal, and gives the result signal displayed like data and settings. Here data means which memory we access like USB flash drive or SD card. 
If there is no USB flash drive and SD card then it is displayed empty option. And if there any one is inserted it shown those options, then we access the internal data. The internal working of all functions is done by the processor. We initially preprogrammed the processor for required functions to access the data [19-20].

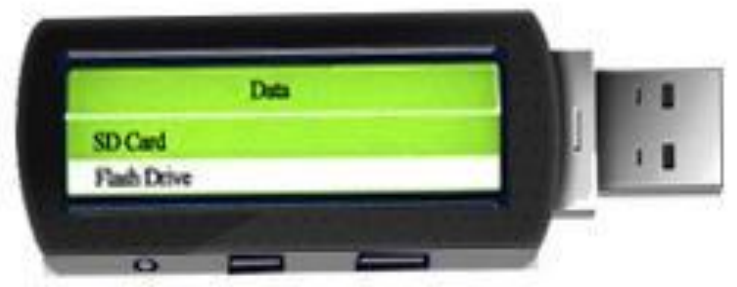

Figure 14. USB Flash Drive Functionality in Dala

Another way of access the data is wireless communication After selecting the menu options then we select settings option two sub options are displayed there are wifi and Bluetooth, and there is ON and OFF options ate shown beside of the We-FI and Bluetooth options. We select the Bluetooth options in built drivers and required hardware is there. Select the Bluetooth option then scan device option is displayed if we select that searching the Bluetooth device for data transfer

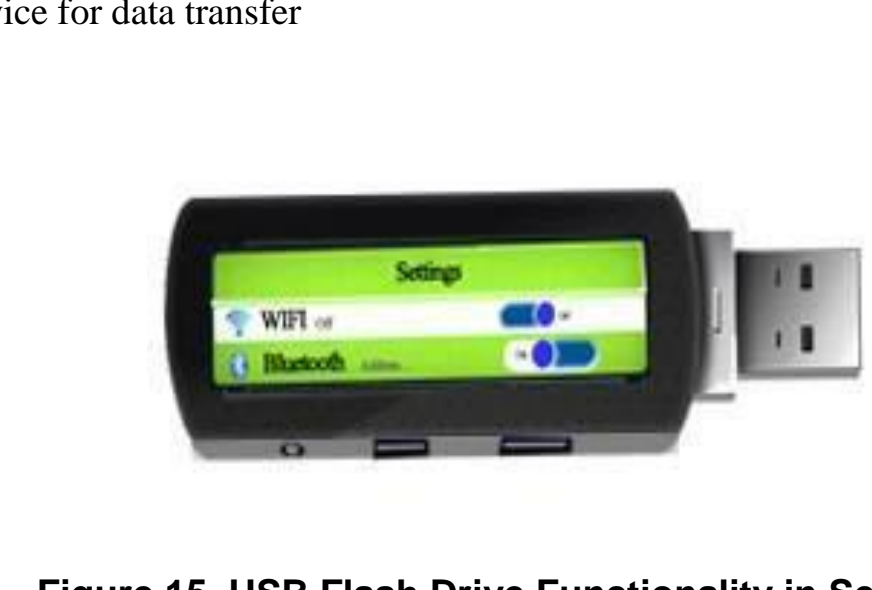

\section{Figure 15. USB Flash Drive Functionality in Settings}

If we select the Wi-Fi option in the settings, then ON and OFF option is there. And another is search option is use to search for the data transmission. Here WI-Fi password is there but we cannot type that password in our LCD. Only open area data can access. 


\section{Data Transmission Flow Chart}

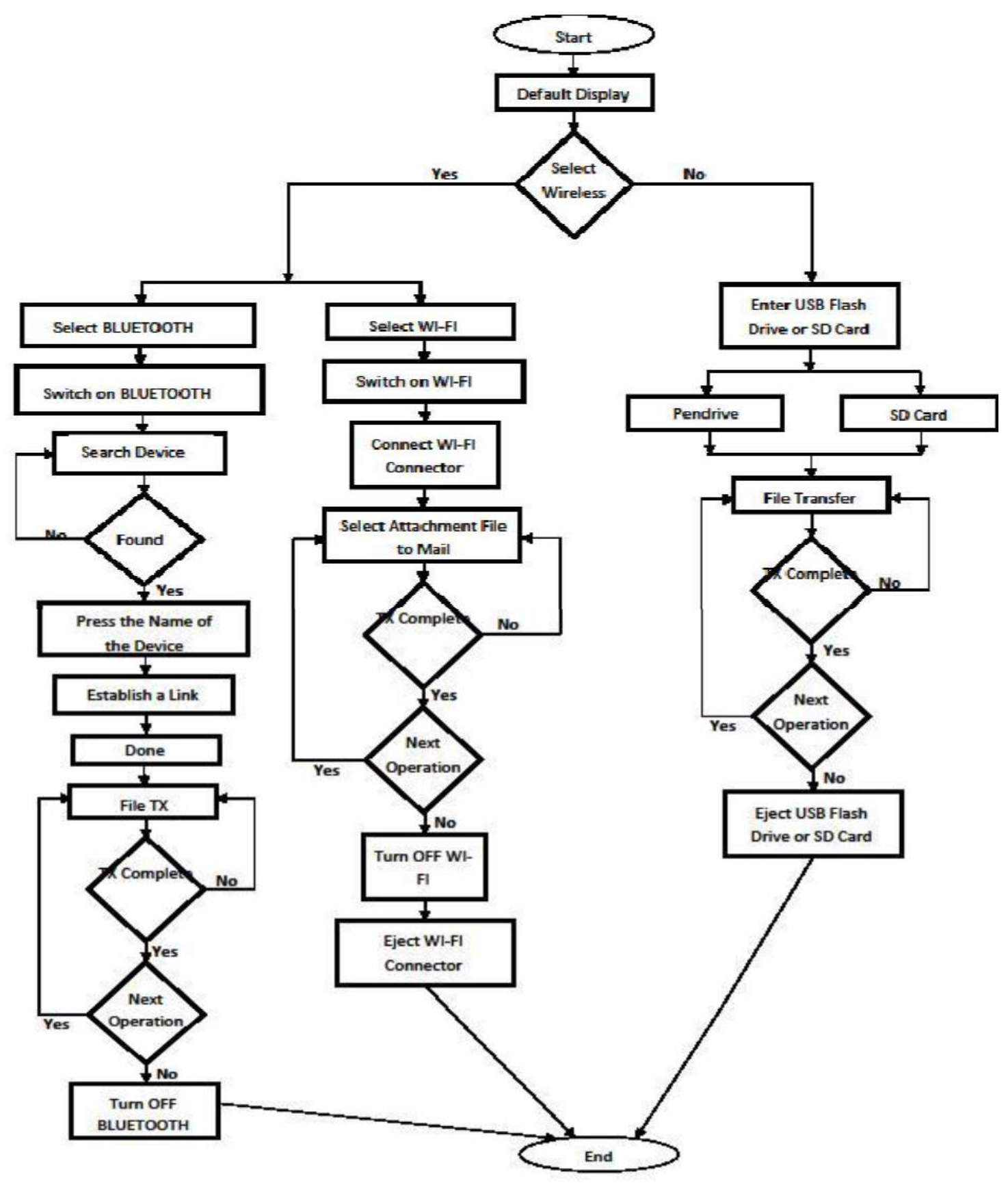

Figure 16. USB Flash Drive Data Transmission Flow Chart

\section{Conclusion}

Data communication between two USB flash drives in today's scenario is the most common task. But the problem is we are not able to share files between them when user is away from computer. We face difficulty when we don't have computer or laptop. It is more 
burdens to purchase a USB data drive than purchasing a laptop or PC. That's way we want to design our idea and create a new touch screen LCD pen drive with not only Wi-Fi and Bluetooth options, and also USB slot and memory card slot. It can use directly pen drive to pen drive data communication without involving PC or laptop. The advantage of this device is no need to extra cable while working for charging, we use internal battery for that, we can charge like our mobile phones and take it away from our house .Currently we have design the system for transfer of data up to 16GB only, but it can be increased by proper selection of the bus.

\section{Acknowledgements}

Authors like to express their thanks to the management of K.L.University for their support and encouragement during this work. Further B. Naresh Kumar Reddy like to express his gratitude towards our Vice-Chancellor Dr. L. S. S. Reddy for their suppørt ln making out this work.

\section{References}

[1] Wireless image transmission based on the Embedded \$ystem by Huo Chunbao, Gao Liduo, Liu Yuwei

[2] Behrouz A Forouzan, "Data Communication and Netwo,rks", 4thedition,

[3] http://en.wikipedia.org/wiki/WiFi.

[4] WiFi: What's Next? Communications Magazine, IEEE, (2002)Decmber.

[5] http://en.kioskea.net/contents/802-introduction-to-wi-fi-802 1 1-or-wifi

[6] http://www.freebsd.org/doc/handbook/network-bluetooth.html

[7] http://members.hellug.gr/nmav/papers/other/Bluetooth\%20security.pdf

[8] Remple, T.B., Qualcomm, San Diego, CA, USA, June 2003 "USB on-the-go interface for portable devices", Consumer Electronics, 20s03.

[9] International Journal of Engineering Research and Development, ISSN: 2278-067X, vol. 1, no. 6, (2012) June, pp. 25-34.

[10] http://en.wikipedia.org/wikj/USB_flash_drive

[11] http://www.absoluteastronomy.com/topics/Universal_Serial_Bus

[12] http://www.easeu com/resource/memory-card -format-recovery.html

[13] http://researchijcaonline.org/Yolume85/numbe r13/pxc3893397.pdf

[14] http://www.8051projects.net/lcd-interfacing/ba sics.php

[15] International Journal of Scientific and Research Publications, vol. 3, Issue (2013), September, ISSN 22503153

[16] P.-C.Huang, Y.-H Chang, T.-W. Kuot, J.-W. Hsieh and M. Lin, "The Behavior Analysis of Flash-Memory Storage Systems, Object Oriented Real-Time Distributed Computing (ISORC), 2008 11th IEEE International Symposium, Print ISBN: 978-0-7695-3132-8 J. Clerk Maxwell, A Treatise on Electricity and Magnetism, 3rded., vol. 2. Oxford: Clarendon, 1892, (2008) May, pp.68-73

[17] http://compnetworking.about.com

[18] Evaluation of Wireless Home Automation Technologies by A. J. Dinusha Rathnayaka, Vidyasagar M. Potdar, Samitha J. Kuruppu in 5th IEEE International Conference on Digital

[19] http//www.indiastudychannel.com/experts/21 785-internal-structure-pen-drive.aspx

[20] http://www.fileguru.com/apps/pen_drive_circu it_diagram. 